\title{
Mecânica Pulmonar de Pacientes em Suporte Ventilatório na Unidade de Terapia Intensiva. Conceitos e Monitorização*
}

\author{
Concepts and Monitoring of Pulmonary Mechanic in Patients under \\ Ventilatory Support in Intensive Care Unit
}

\author{
Eduardo Antonio Faustino ${ }^{1}$
}

\section{RESUMO}

JUSTIFICATIVA E OBJETIVOS: Em ventilação mecânica invasiva e não-invasiva, o conhecimento da fisiologia da mecânica respiratória, é imprescindível para tomada de decisões e no manuseio eficiente dos ventiladores modernos. A monitorização dos parâmetros da mecânica pulmonar é recomendada nos trabalhos de revisão e de pesquisas clínicas. O objetivo deste estudo foi rever os conceitos de mecânica pulmonar e os métodos utilizados para obtenção de medidas à beira do leito, enfatizando três parâmetros: resistência, complacência e PEEP intrínseca.

MÉTODO: Foi realizada revisão bibliográfica através dos bancos de dados LILACS, MedLine e PubMed, no período de 1996 a 2006.

RESULTADOS: Esta revisão abordou os parâmetros de resistência, complacência pulmonar e PEEP intrínseca como fundamentais na compreensão da insuficiência respiratória aguda e suporte ventilatório mecânico, principalmente na doença pulmonar obstrutiva crônica (DPOC) e na síndrome da angústia respiratória aguda (SARA).

CONCLUSÕES: A monitorização da mecânica pulmonar em pacientes sob ventilação mecânica em unidade de

1. Médico Intensivista e Cardiologista da UTI do Hospital de Referência de Gurupi.

*Recebido do Hospital de Referência de Gurupi, Tocantins, TO

- Tese de Conclusão de Pós-Graduação em Ventilação Mecânica

Apresentado em 07 de março de 2007

Aceito para publicação em 22 de maio de 2007

Endereço para correspondência:

Dr. Eduardo Antonio Faustino

Av. Alagoas, 1748 - Gurupi

77410-070 Tocantins, TO

Fones: (63) 3312-4149 / 8121-7952

E-mail: cardiofaustino@bol.com.br

(C)Associação de Medicina Intensiva Brasileira, 2007 terapia intensiva (UTI) pode fornecer dados relevantes e deve ser implementada de forma sistemática e racional.

Unitermos: complacência pulmonar, mecânica pulmonar, mecânica respiratória, monitorização, PEEP intrínseca, resistência pulmonar, ventilação mecânica.

\section{SUMMARY}

BACKGROUND AND OBJECTIVES: In mechanical ventilation, invasive and noninvasive, the knowledge of respiratory mechanic physiology is indispensable to take decisions and into the efficient management of modern ventilators. Monitoring of pulmonary mechanic parameters is been recommended from all the review works and clinical research. The objective of this study was review concepts of pulmonary mechanic and the methods used to obtain measures in the bed side, preparing a rational sequence to obtain this data.

METHODS: It was obtained bibliographic review through data bank LILACS, MedLine and PubMed, from the last ten years.

RESULTS: This review approaches parameters of resistance, pulmonary compliance and intrinsic PEEP as primordial into comprehension of acute respiratory failure and mechanic ventilatory support, mainly in acute respiratory distress syndrome (ARDS) and in chronic obstructive pulmonary disease (COPD).

CONCLUSIONS: Monitoring pulmonary mechanics in patients under mechanical ventilation in intensive care units gives relevant informations and should be implemented in a rational and systematic way.

Key Words: intrinsic PEEP, mechanical ventilation, monitoring, pulmonary compliance, pulmonary mechanic, pulmonary resistance

\section{INTRODUÇÃO}

A ventilação mecânica (VM) é uma das duas principais razões pelas quais um paciente é admitido em UTI de 
acordo com Tobin e col. ${ }^{1}$. O principal objetivo da VM é promover o repouso da musculatura respiratória e reduzir o trabalho da ventilação, expresso em custo de oxigênio. A sincronia paciente-ventilador tem relação direta com o trabalho ventilatório. Para melhor relaxamento e repouso da musculatura inspiratória, o ventilador deverá ciclar em sincronia com a atividade do próprio ritmo respiratório do paciente ${ }^{2}$.

Entretanto, quando é instituída a VM no paciente, muitas variáveis ficam à disposição do intensivista para análise contínua e tomada de decisões. A interpretação desses dados exige conhecimento da fisiologia e mecânica pulmonar. Estudo recente revelou que até $35 \%$ dos residentes não conseguiram selecionar adequadamente um modo ventilatório que reduzisse a PEEP intrínseca em pacientes com DPOC logo após a intubação traqueal ${ }^{3}$.

Outro aspecto relevante é o conceito de lesão pulmonar induzida pelo ventilador, atualmente já bem estabelecido. Até 1998, os conceitos que definiam lesão pulmonar aguda (LPA) e síndrome da angústia respiratória aguda (SARA), não utilizavam fatores relacionados à mecânica pulmonar, mesmo sabendo que a mortalidade poderia ser a mesma para LPA e SARA, a despeito dos valores aplicados no ventilador ${ }^{4}$.

Amato e col. ${ }^{5}$, mostraram que uma forma "protetora" de ventilação, em contraste com uma forma de tratamento com ventilação "normal", reduziu a mortalidade de $71 \%$ para $38 \%$ na SARA, utilizando baixo volumecorrente pulmonar. Apesar de não ter usado dados medidos de mecânica pulmonar, o estudo revelou como os alvéolos colapsados podem ser recrutados baseado no segmento da curva P-V para a complacência pulmonar máxima.

Em 1999, uma ligação entre mecânica pulmonar e morbimortalidade foi suspeitada por Ranieri e Slutsky ${ }^{6}$, relatando que a produção de indicadores inflamatórios e risco de desenvolver falência de múltiplos órgãos era menor com estratégia "protetora". Independentemente da causa, os pulmões lesados têm uma função de barreira anormal. Os capilares pulmonares estão rompidos e as células epiteliais alveolares não podem retirar apropriadamente a água e solutos do espaço alveolar, com importante conseqüência para a mecânica pulmonar. A lesão e o edema aumentam a resistência e a elastância pulmonar?

O colapso de unidades alveolares inundadas e dependentes acarreta aumento na elastância pulmonar refletida pelo menor número de unidades alveolares recrutáveis, não dependentes.
Diante do exposto, o estudo da mecânica pulmonar em pacientes com doença pulmonar obstrutiva crônica (DPOC), revela alterações de resistência das vias aéreas e limitação ao fluxo expiratório, significativos nas tomadas de decisões terapêuticas para combater a hiperinsuflação dinâmica própria dessa condição, diminuindo o trabalho imposto à inspiração e favorecendo o desmame da VM.

O objetivo deste estudo foi descrever e analisar os conceitos de mecânica pulmonar envolvidos nas doenças respiratórias prevalentes em UTI, buscando obter benefícios que uma monitorização racional da mecânica respiratória possa trazer no seguimento do paciente sob ventilação mecânica invasiva (VMI).

\section{MÉTODO}

Buscou-se referências bibliográficas nos bancos de dados LILACS, MedLine e PubMed nos últimos 10 anos (1996-2006), usando os unitermos respiratory, pulmona$r y$, mechanics e PEEP. Os trabalhos experimentais pioneiros no estudo da mecânica pulmonar foram realizados na primeira metade do século $X X$, nas décadas de 1950 e 1960, podendo-se alguns serem averiguados a partir de referências de estudos mais recentes.

\section{RESULTADOS}

Os estudos e artigos revistos salientam a necessidade de se obter as medidas de mecânica pulmonar através de métodos rigorosos e consagrados. A análise das curvas de pressão, fluxo e volume nas telas dos ventiladores modernos, associada a estudos que utilizaram videomicroscopia direta de alvéolos subpleurais e estudos com tomografia computadorizada puderam correlacionar a dinâmica alveolar com as alterações regionais dos volumes pulmonares, as alterações na função do surfactante e a presença de hiperinsuflação pulmonar dinâmica . A técnica da oclusão inspiratória final para medir as resistências foi diversificada e adaptada aos ventiladores contemporâneos, a decomposição da mecânica pulmonar e da caixa torácica em vários estudos, mostraram o comportamento destes dois componentes, em situações clínicas e cirúrgicas diversas.

\section{DISCUSSÃO}

As propriedades mecânicas dinâmicas do sistema respiratório dizem respeito a situações em que o flu- 
xo varia ao longo do tempo, enquanto as propriedades estáticas realçam a ausência de fluxo ${ }^{8}$. O trabalho mecânico dos pulmões na respiração pode ser obtido confrontando-se em um gráfico o volume e a pressão transpulmonar. A área resultante volume-pressão inclui um componente estático e um dinâmico. O primeiro reflete o trabalho devido às propriedades mecânicas estáticas e o segundo reflete as variáveis dinâmicas, ou seja a resistência intrínseca das vias aéreas e a resistência adicional que é devida às desigualdades nas constantes de tempo de enchimento e esvaziamento das unidades alveolares e propriedades viscoelásticas teciduais $^{9}$

A decomposição das resistências pulmonares foi inicialmente descrita por Von Neergard em 1927, quando descreveu dois métodos para medir a resistência ao fluxo: o método interruptor e as subtrações elásticas, que permitem decompor a resistência pulmonar (Rpulm) em resistências intrínseca e adicional.

Bates e col. ${ }^{10}$, utilizando o método interruptor, mostraram que à oclusão súbita das vias aéreas ao final da inspiração segue-se à diminuição imediata na pressão transpulmonar de valor máximo, que é a pressão de pico ou Pmáx, para um valor definido como P1. Dividindo-se a diferença Pmáx - P1 pelo fluxo imediatamente precedente à oclusão se obtém as resistências estáticas denominada de Rmin ou Rinicial, que reflete somente a resistência das vias aéreas. Experimentos em animais mostraram que ela aumenta linearmente com o fluxo, devido este ser não laminar nas grandes vias aéreas.

Mantendo-se a oclusão, segue-se lenta e gradual diminuição da pressão até atingir um valor de platô, P2, que representa a pressão de recolhimento elástico inspiratório final estática do pulmão. Dividindo a diferença P1 - P2 pelo fluxo precedente têm-se a resistência adicional ( $\mathrm{R}$ adic), porém neste momento de diminuição lenta, inexiste fluxo, ou seja, esta resistência não reflete um componente resistivo puro e por isso deixa de ser importante no cálculo da resistência pulmonar total. O tempo decorrido entre P1 e P2 é necessário para a acomodação do ar nos pulmões o que ocorre por dois fenômenos: Pendelluft, que é a transferência de pequenos volumes de gás de alvéolos com altas pressões para alvéolos com baixas pressões, devido às desigualdades nas constantes de tempo das unidades alveolares e o relaxamento de tensão do tecido pulmonar - stress relaxation ${ }^{11}$.

Quanto à complacência, é importante entender a diferença entre complacência estática (Cst) e compla- cência dinâmica (Cdyn) do sistema respiratório. Para a complacência estática a variação de volume refere-se à pressão de platô estática (P2). Para a complacência dinâmica a variação de volume refere-se à pressão de pico inspiratória - Ppico .

Para a estimativa da resistência e da complacência do sistema respiratório, o paciente deve estar sedado e paralisado, sem atividade muscular, em ventilação controlada a volume, fluxo constante (onda quadrada) com a técnica de oclusão ao final da inspiração ${ }^{10}$. Importante lembrar que, quando a pausa de oclusão inspiratória for menor que dois segundos, a pressão de platô não refletirá a pressão alveolar quando analisada em pacientes com doença pulmonar grave (DPOC e SARA). Portanto, as manobras de oclusão devem durar cerca de 4 segundos ${ }^{12}$.

A análise da complacência e da resistência pulmonar a partir da curva $\mathrm{P}-\mathrm{V}$ utiliza métodos estáticos e quasiestáticos. Os métodos estáticos, técnicas da superseringa e da oclusão inspiratória, mostraram ser fidedignos, porém desvantajosos, por necessitarem desconexão do paciente da prótese ventilatória, além da duração demorada do procedimento e da perda de volume pulmonar por absorção de oxigênio. O método quasiestático necessita insuflação contínua de fluxos constantes, gerando resistência pelas conexões e vias aéreas, que deve ser subtraída da pressão total encontrada ou então utilizar fluxos muito baixos. Estudos mostraram que o fator resistivo imposto por altos fluxos pode não ser clinicamente relevante, se forem usados baixos fluxos (menores que $9 \mathrm{~L} / \mathrm{min}$ ), tornando este método de fácil execução à beira de leito nos ventiladores modernos com telas de curvas (em torno de 2 min para sua realização).

Em ventilações espontâneas, a complacência total do sistema respiratório, incluindo parede torácica e pulmões são de $100 \mathrm{~mL} / \mathrm{cmH}_{2} \mathrm{O}$. A complacência pulmonar está ao redor de $200 \mathrm{~mL} / \mathrm{cmH}_{2} \mathrm{O}$ e em indivíduos normais anestesiados e ventilados é de $70-80 \mathrm{~mL} /$ $\mathrm{cmH}_{2} \mathrm{O}$. Um pulmão rígido como nos pacientes com SARA tem baixa complacência, enquanto um pulmão altamente distensível como no enfisema pulmonar tem complacência muito alta. No indivíduo normal o ponto de inflexão superior da curva P-V ocorre em volume pulmonar três litros acima da capacidade residual funcional (CRF), o qual define a capacidade pulmonar total, e corresponde à pressão acima da qual inicia-se a hiperdistensão pulmonar ${ }^{13}$.

Algumas vezes o ponto de inflexão inferior da curva $\mathrm{P}-\mathrm{V}$ pode não ser identificado em pacientes com insufi- 
ciência respiratória aguda. Vieira e col. ${ }^{14}$ mostraram que estes pacientes eram mais jovens, a complacência do sistema respiratório mais baixo e a mortalidade tendia a ser mais alta. Nestes pacientes as opacidades radiográficas predominavam nos lobos inferiores, as atenuações à tomografia computadorizada se aproximavam aquelas da água (7 HU) ou seja, regiões não aeradas, e a PEEP induziu recrutamento alveolar que esteve associado com hiperdistensão pulmonar.

Os fisiologistas, em seus estudos pioneiros, afirmaram que a insuflação e a desinsuflação pulmonar resultavam da soma das expansões e contrações uniformes individuais de cerca de 300 milhões de alvéolos. A videomicroscopia direta em pulmões de animais avaliou as mudanças no volume pulmonar e essas ocorriam muito mais por recrutamento e desrecrutamento alveolar do que por mudanças uniformes simultâneas no volume alveolar, e que a insuflação até $80 \%$ da capacidade pulmonar total (CPT) acarretava pouca mudança no volume alveolar, mas um significativo aumento no número de alvéolos recrutados $^{15}$.

A hiperinsuflação de alvéolos normais e abertura/fechamento cíclicos de alvéolos colapsados contribuem para a lesão pulmonar progressiva não somente pelo processo da doença em si, mas também, como resultado de padrões ventilatórios aplicados durante o curso da doença. A ruptura alveolar com extravasamento de ar (barotrauma), a hiperdistensão alveolar (volutrau$\mathrm{ma}$ ), recrutamento/desrecrutamento de alvéolos colapsados (atelectrauma) e mesmo lesão sutil manifestada pela ativação de processo inflamatório (biotrauma), compõem o espectro da lesão induzida pela ventilação (VILI).

Terragni e Ranieri ${ }^{16}$, sugerem um parâmetro de monitorização que denominam "índice de estresse" (stress index), utilizando o perfil da curva pressão-tempo para detectar e/ou prevenir VILI em pacientes com SARA. Se a curva de pressão apresenta concavidade para cima sinaliza redução da complacência pulmonar (índice $>1$ ), se a concavidade é para baixo mostra que a complacência aumentou (índice $<1$ ) e se igual a 1 a complacência permanece constante. Vários estudos em animais e humanos evidenciaram boa correlação com melhores valores de elastância, níveis de citocinas e sinais morfológicos de lesão, quando ajustes nos modos ventilatórios permitissem obter índices de estresse igual a $1^{16}$.

$\mathrm{Na}$ VILI, contudo, o volutrauma é o único mecanismo até agora confirmado em estudos clínicos ${ }^{17}$. A partir de estudos de análise do volume pulmonar a nível regional, tem-se contestado a existência do mecanismo de recrutamento/desrecrutamento na gênese da VILI, baseado em modelo de lesão pulmonar no qual o edema alveolar mais do que o colapso é o achado patológico predominante ${ }^{18-20}$.

DiRocco e col. ${ }^{17}$ realizaram estudos em ratos com videomicroscopia e cortes histológicos, provocando lesão pulmonar aguda com ácido oléico, detergente, barotrauma e endotoxina bacteriana. Em todos os modelos, a quantidade de água nos alvéolos era maior que nos grupos controles. Com exceção do grupo que recebeu endotoxina os outros grupos mostraram alvéolos mais instáveis, colapsando e expandindo a cada ventilação, enquanto no grupo endotoxina os alvéolos perderam a sua forma, com aparência sólida e branca, mas permaneceram estáveis durante a ventilação. Concluíram que a disfunção de surfactante pulmonar era a causa mais provável para o desenvolvimento da instabilidade alveolar, podendo não estar presente em todos os pacientes com SARA e nem a oxigenação refletir o grau de lesão prevalente.

Anatomicamente os alvéolos são unidades interdependentes com integridade estrutural que lembra um favo de mel, quando combinadas com as propriedades do surfactante de diminuir a tensão superficial, fornecem estabilidade física ao alvéolo, resultando em pouca mudança no volume alveolar durante a ventilação. Gatto e col..21, utilizaram videomicroscopia direta de alvéolos subpleurais e mostraram a não variabilidade no volume e no tamanho de alvéolos normais com a ventilação, mesmo em condições de alta pressão nas vias aéreas. Entretanto, nos alvéolos com função de surfactante alterada desenvolvia-se uma amplitude variável de anormalidades da mecânica alveolar, que denominaram colapso e expansão alveolar repetidos. Alguns alvéolos colapsavam parcialmente, enquanto outros o faziam completamente para logo em seguida se re-expandirem. Porém, o colapso de apenas um alvéolo no ácino ocasionava estresse por cisalhamento (shear stress) não somente do alvéolo colapsado ou hiper-distendido, como também distorcia os alvéolos adjacentes desencadeando pressões tão altas quanto $140 \mathrm{cmH}_{2} \mathrm{O}$.

Os conhecimentos atuais sobre mecânica no pulmão edemaciado, baseiam-se em interpretações de tomografias computadorizadas e análises das curvas P-V. Inferiu-se a partir daí, que regiões dependentes de pulmões edemaciados colapsavam sob baixas pressões transpulmonares e reabriam abruptamente em pres- 
sões de abertura elevadas. Wilson e col. ${ }^{19}$, mediram o volume de regiões dependentes de pulmões de cães lesados por ácido oléico, revelando que este volume regional era maior do que aquele de pulmões normais, porém, o volume-corrente era menor, o que fala contra o conceito pré-estabelecido de que regiões dependentes estão colapsadas em pulmões edematosos. Nos pulmões edemaciados, pequena quantidade de fluido fica confinado nos cantos do interior dos alvéolos, enquanto grande quantidade de fluido se estende para as bordas livres das paredes alveolares curvando levemente as interfaces ar-líquido. Ao fornecer baixos volume e pressão, o fluido se abaula em direção ao ducto alveolar adjacente comprimindo a entrada do alvéolo. À medida que o volume aumenta, a bolha de ar é forçada para dentro do alvéolo aumentando rapidamente a tensão e a área de superfície, ainda que neste ponto a complacência mantenha-se pequena. Com volumes progressivamente maiores a curva $\mathrm{P}-\mathrm{V}$ torna-se quase que vertical refletindo ganho na complacência.

No indivíduo sadio, ao final de uma expiração normal, a pressão alveolar aproxima-se de zero. Durante uma expiração passiva, a taxa de esvaziamento depende da constante de tempo expiratória do sistema, isto é, do produto da complacência total pela resistência total. A fim de alcançar expiração passiva quase total (99\%) o sistema respiratório exige um tempo expiratório igual a 5 vezes a sua constante de tempo expiratória ao passo que um tempo expiratório de 3 vezes a constante de tempo permite $95 \%$ de expiração total. Ocorrerá limitação ao fluxo expiratório quando a resistência total e/ou a complacência total forem altas, a freqüência respiratória rápida e o tempo expiratório curto, o que impedirá o esvaziamento completo do pulmão.

Esta impossibilidade do pulmão retornar à sua capacidade residual funcional (volume pulmonar ao final de uma expiração passiva) caracteriza a hiperinsuflação pulmonar. O aumento do volume pulmonar acima da CRF em pacientes em VM, tem como causa mais comum a aplicação de PEEP externa, ajustada no apareIho de ventilação e denomina-se hiperinsuflação pulmonar estática. A hiperinsuflação pulmonar dinâmica ocorre quando uma nova inspiração inicia-se antes que a expiração total seja completada, ocasionando aumento no volume expiratório final por motivos dinâmicos e não por interferência externa (estática), aplicada no ventilador ${ }^{12}$.

O volume expiratório final na hiperinsuflação pulmonar dinâmica representa uma PEEP total, que se descontar a PEEP externa ajustada do ventilador, será encontrada a PEEP intrínseca ou auto-PEEP, que é a PEEP gerada pela hiperinsuflação dinâmica. A auto-PEEP é uma carga adicional a ser superada pelo ventilador durante a ventilação passiva. A PEEP intrínseca ou auto-PEEP, deve ser levada em consideração a fim de se obter valor correto para a complacência estática do sistema respiratório.

Também para medir a PEEP total e conseqüentemente a PEEPi (PEEPi = PEEPtotal - PEEPext), é necessário manobra de oclusão, agora ao final da expiração. A oclusão deverá ser mantida por cerca de 4 segundos para permitir equilíbrio pressórico intrapulmonar, certificando-se que não há atividade muscular expiratória. A aferição da PEEPi em pacientes respirando ativamente é mais incerta, porém pode ser realizada e necessita a instalação de um balão esofágico para medida da pressão esofágica (pressão intrapleural).

Em pacientes, com limitação ao fluxo expiratório e colapso dinâmico das vias aéreas, a contração da musculatura expiratória agravará o quadro de limitação ao fluxo, movendo o ponto de igual pressão para mais próximo dos alvéolos. Nesta situação não há como medir a auto-PEEP com precisão e o uso da PEEP externa não é recomendado pelo esforço adicional imposto ao sistema respiratório.

A abordagem da mecânica respiratória é mandatória para avaliar a gravidade da doença, otimizar os ajustes no ventilador e analisar os efeitos das várias modalidades terapêuticas. Na prática clínica corrente, as aferições são limitadas ao cálculo da complacência estática (Cst), da resistência máxima (Rmáx) e da pressão expiratória final positiva intrínseca (PEEPi), para o sistema respiratório completo ${ }^{12}$.

O ideal é iniciar as medidas logo após a intubação traqueal, momento em que o paciente encontra-se profundamente sedado e curarizado. A rápida diminuição pressórica inicial corresponde à dissipação pressórica nas vias aéreas e é especialmente importante avaliá-la nos pacientes com DPOC e SARA, nos quais a resistência das vias aéreas está aumentada.

Antonaglia e col.22, estudaram o comportamento viscoelástico do sistema respiratório, responsável pela maior parte da pressão dissipada durante a ventilação em indivíduos normais e com lesão pulmonar aguda (LPA). A técnica da oclusão inspiratória final rápida revela um modelo viscoelástico linear com descrição acurada tempo-dependente da resistência e da elastância do sistema respiratório; entretanto, a obtenção desses valores exige múltiplas insuflações isovolúmicas com diferentes fluxos inspiratórios ou múltiplas 
insuflações com o mesmo fluxo e diferentes volumes. O método preconizado por eles utiliza apenas duas oclusões inspiratórias finais, uma após tempo inspiratório curto para determinar o componente elástico da viscoelasticidade e a outra com um tempo inspiratório longo, para avaliar o componente resistivo da viscoelasticidade. Nos pacientes normais, quanto menor o tempo inspiratório em relação à constante de tempo (R/E ou $\mathrm{RxC}$ ), a elastância adicional melhor representa a elastância total, e quanto mais longo o tempo inspiratório em relação à constante de tempo, a resistência adicional melhor representa a resistência total do sistema respiratório. Nos pacientes com lesão pulmonar aguda o método fornece dados semelhantes com razoável acurácia em comparação com o método de múltiplas insuflações.

O sistema respiratório pode ser decomposto em dois componentes:a parede torácica e os pulmões. O diafragma está mecanicamente acoplado à parede e conteúdo abdominais. Qualquer aumento na pressão abdominal pode afetar a mecânica pulmonar, aumentando a propensão para o desenvolvimento de atelectasias e diminuindo a capacidade residual funcional (CRF). Pode também afetar diretamente a mecânica da parede torácica alterando a sua configuração, ou causando mudanças nas zonas de interação nas quais os pulmões se apõem à superfície lateral do diafragma. Algumas situações clínicas podem, também, levar a aumentos na pressão abdominal por distensão, como ascite hipertensiva, hemorragias abdominais, obstrução intestinal, laparoscopia, grandes tumores e diálise peritoneal. Contudo, não se sabe se apenas edema de alças intestinais e íleo podem afetar a mecânica respiratória. Ranieri e col. ${ }^{23}$, compararam pacientes com SARA de origem clínica e após cirurgias abdominais de grande porte, avaliando mudanças nas pressões intratorácicas e intra-abdominais através da pressão esofágica (Pes) e gástrica (Pga). Estudos prévios em animais já haviam chamado atenção para o efeito deletério da distensão abdominal na mecânica respiratória através da repercussão sobre a parede torácica. Os pacientes do grupo de grande cirurgia abdominal com distensão abdominal significativa mostraram achatamento e convexidade para cima da curva P-V do sistema respiratório e dos pulmões, e progressiva diminuição da complacência ao aumento de volume-corrente insuflado. Em contraste, os pacientes com SARA de origem clínica, as curvas P-V exibiam concavidade para cima e progressivo aumento na complacência. Nos pacientes com distensão abdominal o volume-corrente com o qual a complacência começa a diminuir é em média $28 \%$ maior quando a curva $\mathrm{P}-\mathrm{V}$ do pulmão é comparada com a curva do sistema respiratório total; entretanto, na prática clínica é a curva do sistema respiratório total que é determinada rotineiramente, levando potencialmente a uma subestimação do volume-corrente tolerável. O estudo concluiu que o ponto de inflexão inferior da curva P-V deverá ser $25 \%$ a $30 \%$ mais alto do que aquele calculado com base na mecânica pulmonar. $\mathrm{Na}$ estratégia protetora com utilização de baixos volumescorrentes, a análise da mecânica baseada na curva P$\checkmark$ do sistema respiratório pode levar ao uso de níveis de PEEP muito altos e níveis de volume-corrente mais baixos ocasionando níveis mais altos de $\mathrm{PaCO}_{2}$. Musch e col. ${ }^{24}$, mostraram que a complacência diminuída da parede torácica pode ocorrer por distensão abdominal secundário à hipomotilidade colônica, rigidez de parede torácica agravada por anestésicos (fentanil), e edema de parede torácica por sobrecarga hídrica. Para a complacência pulmonar, podem diminuí-la os derrames pleurais e infiltrados pneumônicos. A resistência da parede torácica não é um componente significativo na resistência pulmonar mais alta dos pacientes com DPOC, porém ela é mais alta nos pacientes com DPOC em relação aos normais. As propriedades reológicas da parede torácica podem ser anormais nas doenças restritivas do tórax e também na DPOC exacerbada ou descompensada.

Ranieri e col. ${ }^{25}$, compararam indivíduos normais e pacientes com insuficiência respiratória aguda na DPOC, revelando a pequena contribuição da parede torácica para a PEEP intrínseca do sistema respiratório total, e que PEEP extrínsecas muito altas $\left(15 \mathrm{cmH}_{2} \mathrm{O}\right)$ agravaram as propriedades elásticas do pulmão e parede torácica. Pacientes submetidos à pneumectomia para cirurgia de redução no volume pulmonar ${ }^{26}$ mostraram diminuição da resistência pulmonar sem alterações nas propriedades mecânicas da parede torácica. Houve diminuição do componente elástico da resistência sem alteração do componente inicial resistivo, evidenciando a importância das desigualdades nas constantes de tempo do tecido pulmonar que ocorrem no DPOC. A diminuição do volume pulmonar e a conseqüente diminuição da resistência adicional estiveram associadas com redução da dispnéia depois da cirurgia. Também ocorreu aumento na complacência dinâmica como resultado da diminuição na pressão elástica adicional armazenada nas unidades viscoelásticas do tecido pulmonar. O pulmão esvazia melhor e a limitação ao fluxo expiratório é menor, dessa forma os pacientes não pre- 
cisam mais aumentar o seu volume pulmonar para meIhorar o fluxo expiratório. Além disso, como o volume expiratório final fica menor, os músculos expiratórios operam numa posição mais favorável da curva tensão-comprimento melhorando a capacidade de gerar pressão. Acrescente-se a tudo isto redução no colapso dinâmico contribuindo para melhora sintomática, pois a compressão das vias aéreas colabora para a dispnéia. Nava e col. ${ }^{27}$, estudaram pacientes com fibrose pulmonar terminal em lista de espera para transplante, fornecendo pela primeira vez informações acerca da separação das propriedades resistivas e elásticas do sistema respiratório total entre as mecânicas do pulmão e parede torácica. A diminuição significativa da complacência pulmonar chegou a ser maior comparando com pacientes que não sobreviveram na SARA. A complacência da parede torácica respondeu somente por cerca de $15 \%$ da complacência total do sistema respiratório. A resistência esteve significativamente aumentada, porém em menor magnitude do que nos pacientes com DPOC, sendo a resistência pulmonar o componente mais importante com aumento de ambas resistências interruptora e adicional. O que explica estes resultados é a característica da doença nos estágios finais com achados patológicos em mosaico, isto é, áreas com fibrose mais intensa (subpleurais) e áreas com atividade inflamatória mais ativa. Nos estágios finais, os pulmões tornam-se tão duros e a resistência aumenta a tal ponto, que os músculos respiratórios não sustentam mais a carga de trabalho.

Os pacientes com grave obstrução nas vias aéreas comumente exibem limitação ao fluxo expiratório em respiração normal, particularmente em insuficiência respiratória aguda. O fluxo expiratório é máximo durante a respiração em repouso, portanto em doença grave a ocorrência de limitação ao fluxo expiratório é crítica e conseqüentemente ocorrerá hiperinsuflação pulmonar dinâmica. A hiperinsuflação pulmonar dinâmica não está restrita aos pacientes com DPOC, mas pode também ocorrer nos pacientes com SARA, da mesma forma apresentando aumento na resistência pulmonar ${ }^{11}$. Além disso, deve-se levar em consideração, o aumento na resistência imposta por tubos traqueais estreitos e o acúmulo de secreções nas vias aéreas.

A limitação ao fluxo expiratório e hiperinsuflação pulmonar dinâmica podem ser detectados no gráfico da curva fluxo-volume com o método da pressão expiratória negativa (NEP), aplicando-se pressão negativa de $5 \mathrm{cmH}_{2} \mathrm{O}$ na abertura da via aérea no início da expiração ${ }^{28}$. Nos pacientes que não apresentam aumento da resistência com limitação ao fluxo aéreo, ocorre aumento do fluxo ao longo da expiração, enquanto que naqueles que apresentam limitação ocorre aumento apenas no início, no restante da expiração é semelhante ao fluxo da respiração controle ${ }^{29,30}$.

Nos gráficos de pressão, fluxo e volume, a PEEPi pode ser detectada após rápida oclusão no ramo expiratório do ventilador, permitindo um intervalo para o equilíbrio entre as pressões das vias aéreas e alveolares. Na presença de PEEPi, a pressão na via aérea aumenta e o valor de platô revelado traduz o nível da PEEPi. No gráfico da alça fluxo-volume, a PEEPi é detectada ao final da expiração, onde a alça expiratória aparece truncada, não retornando à linha de base ${ }^{29}$. O efeito da resistência aumentada é somente visualizado na alça expiratória da curva fluxo-volume pela diminuição no pico de fluxo expiratório, sem afetar o padrão de fluxo inspiratório, além de ser particularmente importante na avaliação à resposta medicamentosa broncodilatadora ${ }^{8}$.

A diferença entre o volume pulmonar expiratório final e o volume residual, é definido como $\triangle \mathrm{CRF}$ e pode ser obtido prolongando-se o tempo expiratório e permitindo que o paciente exale até alcançar o volume residual (Vr). Em alguns pacientes com DPOC o tempo expiratório pode ser tão longo quanto 40 segundos, até alcançar o $\mathrm{Vr}$ e quantificar a hiperinsuflação dinâmica com o $\triangle \mathrm{CRF}$. Em pacientes sob VM assistido-controlada ou pressão-suporte, a PEEPi aumenta os esforços inspiratórios necessários para disparar o ventilador e pode levar a esforços inspiratórios crescentes com repercussão hemodinâmica, fadiga muscular inspiratória e assincronia paciente-ventilador, além de explicar as falhas nas tentativas de desmame. A monitorização da hiperinsuflação pulmonar dinâmica permite limitar a ocorrência de volutrauma e hiperdistensão alveolar ${ }^{11}$.

Ainda que a SARA seja descrita como uma síndrome de baixa complacência, também a resistência das vias aéreas pode estar aumentada como já referido. Junto à possibilidade de desenvolvimento de SARA na presença de asma ou doença pulmonar obstrutiva crônica, um leve aumento na resistência é encontrado nos pacientes com LPA/SARA, independentemente da doença de vias aéreas pré-existente. $O$ aumento da Rsr é essencialmente devido a redução no volume pulmonar aerado, o qual em troca modifica as propriedades viscoelásticas da árvore traqueobrônquica e na maioria dos pacientes não há broncoconstrição verdadeira ${ }^{13}$. No início da SARA, a análise das curvas de pressão e volume ao término da manobra de oclusão inspiratória 
final das vias aéreas, pode revelar que o componente resistivo (ohmic, Pressão de pico - P1), está aumentado em proporção inversa à diminuição da capacidade residual funcional, sugerindo que este não é devido ao estreitamento das vias aéreas mas a um menor volume no pulmão ventilado. A resistência adicional (P1 - P2) está aumentada em todos os pacientes indicando as desigualdades nas constantes de tempo do pulmão doente.

Baixos níveis de PEEPe podem diminuir a resistência média das vias aéreas ao normalizar o componente resistivo inicial, provavelmente através de um aumento do volume pulmonar. Entretanto PEEPe mais altas (aproximadamente $15 \mathrm{cmH}_{2} \mathrm{O}$ ) aumentam a resistência total, quase que exclusivamente através de um aumento no componente adicional, conseqüentemente aumentando as desigualdades regionais sob grandes volumes pulmonares. Deve-se estar atento à possibilidade de desenvolvimento de PEEPi com o uso de baixo volume-corrente (estratégia protetora) pois para manter a ventilação-minuto acaba-se utilizando freqüências respiratórias mais altas. Neste caso a PEEPi pode estar mais alta do que a PEEP ajustada no ventilador ${ }^{31}$.

Em pacientes com SARA a análise da curva $P-V$, que apresenta perfil sigmóide, é útil pois permite o ajuste apropriado da PEEP e do volume-corrente (Vt) no ventilador. Nela, o ponto de inflexão inferior corresponde a pressão de abertura na maioria das vias aéreas pequenas previamente colapsadas e recrutadas durante a $1^{\text {a }}$ parte da curva $\mathrm{P}-\mathrm{V}$. A porção linear da curva corresponde ao momento em que todas as unidades alveolares estão abertas e a complacência é máxima. $O$ ponto de inflexão superior delimita o momento a partir do qual as unidades alveolares cheias de ar tornam-se hiperdistendidas. O recrutamento alveolar é um fenômeno progressivo ocorrendo durante a insuflação do volume-corrente, quando induzido pela PEEP é maior com baixos volumes-correntes $(6 \mathrm{~mL} / \mathrm{kg})$ do que com grandes volumes-correntes $(12 \mathrm{~mL} / \mathrm{kg})$.

\section{CONCLUSÃO}

O avanço tecnológico dos ventiladores modernos e os métodos fisiológicos de estudo da mecânica respiratória facilitaram a monitorização dos parâmetros de resistência e complacência pulmonar. Possibilitou, com a técnica de oclusão das vias aéreas, obter medidas estáticas ou quase-estáticas da curva pressão-volume do sistema respiratório.

A quantificação da PEEP intrínseca, associada ou não à limitação do fluxo expiratório, é útil nos pacientes com DPOC nos com SARA com menor freqüência, além de orientar a utilização de PEEP externa na sincronia paciente-ventilador e no processo de desmame.

A técnica de oclusão inspiratória final, permite separar as resistências das vias aéreas da resistência tecidual, e se associada ao balão esofágico decompor as resistências do pulmão e caixa torácica.

Na SARA, a análise da curva pressão-volume revela os diferentes padrões evolutivos da complacência e da recrutamento alveolar. O potencial de recrutamento na fase inicial exsudativa com complacência diminuída e a seguir próxima do normal; e na fase tardia, fibroproliferativa, de baixa complacência e pouco potencial de recrutamento.

No entanto, constatou-se que nos estudos realizados envolvendo a necessidade da obtenção de dados de mecânica respiratória, utilizaram-se várias medidas de PEEP externa de maneira aleatória. Cabe perguntar se a padronização de um único valor de PEEP externa aplicada, refletiria melhor e mais fidedignamente os valores dos parâmetros da mecânica pulmonar .

\section{REFERÊNCIAS}

01. Tobin MJ - Principles and Practice of Mechanical Ventilation $2^{\text {nd }}$ Ed, McGraw Hill. 2006.

02. Tobin MJ, Jubran A, Laghi F - Patient-ventilator interaction. Am J Respir Crit Care Med, 2001;163:1059-1063.

03. Cox CE, Carson SS, Ely EW et al - Effectiveness of medical resident education in mechanical ventilation. Am J Respir Crit Care Med, 2003;167:32-38.

04. Stenqvist O - Practical assessment of respiratory mechanics. Br J Anaesth, 2003;91:92-105.

05. Amato MB, Barbas CSV, Medeiros D et al - Effect of a protective-ventilation strategy on mortality in the acute respiratory distress syndrome. $\mathrm{N}$ Engl J Med, 1998;338:347-354.

06. Ranieri VM, Suter PM, Tortorella $\mathrm{C}$ et al - Effect of mechanical ventilation on inflammatory mediators in patients with acute respiratory distress syndrome: a randomized controlled trial. JAMA, 1999;282:54-61.

07. de Chazal I, Hubmayr RD - Novel aspects of pulmonary mechanics in intensive care. Br J Anaesth, 2003;91:81-91.

08. Lucangelo $U$, Bernabe F, Blanch $L$ - Respiratory mechanics derived form signals in the ventilator circuit. Respir Care, 2005;50:55-67.

09. Milic-Emili J - Pulmonary flow resistance. Lung, 1989;167:141-148.

10. Bates $\mathrm{JH}$, Rossi A, Milic-Emili $\mathrm{J}$ - Analysis of the behavior of the respiratory system with constant inspiratory flow. J Appl Physiol, 1985;58:18401848.

11. Guerin C, Tantucci C - Respiratory Mechanics in Intensive Care Units, em: Milic-Emili J - The European Respiratory Monography, European Respiratory Society Journals, Ltd., 1999;255.

12. Iotti GA, Braschi A - Monitorização da Mecânica Respiratória. Editora Atheneu, 2006.

13. Lu Q, Rouby JJ - Measurement of pressure-volume curves in patients on mechanical ventilation: methods and significance. Crit Care, 2000;4:91-100.

14. Vieira SR, Puybasset L, Lu Q et al - A scanographic assessment of pulmonary morphology in acute lung injury. Significance of the lower inflection point detected on the lung pressure-volume curve. Am J Respir Crit Care Med, 1999;159:1612-1623. 


\section{MECÂNICA PULMONAR DE PACIENTES EM SUPORTE VENTILATÓRIO NA UNIDADE DE TERAPIA INTENSIVA. CONCEITOS E MONITORIZAÇÃO}

15. Carney DE, Bredenberg CE, Schiller HJ et al - The mechanism of lung volume change during mechanical ventilation. Am J Respir Crit Care Med, 1999;160:1697-1702.

16. Terragni PP, Rosboch GL, Lisi A et al - How respiratory system mechanics may help in minimising ventilator-induced lung injury in ARDS patients. Eur Respir J, 2003;22:(Suppl42):15S-21S.

17. DiRocco JD, Pavone LA, Carney DE et al - Dynamic alveolar mechanics in four models of lung injury. Intensive Care Med, 2006;32:140-148.

18. Martynowicz MA, Minor TA, Walters BJ et al - Regional expansion of oleic acid-injured lungs. Am J Respir Crit Care Med, 1999;160:250-258.

19. Wilson TA, Anafi RC, Hubmayr RD - Mechanics of edematous lungs. Appl Physiol, 2001;90:2088-2093.

20. Hubmayr RD - Perspective on lung injury and recruitment: a skeptical look at the opening and collapse story. Am J Respir Crit Care Med, 2002;165:1647-1653.

21. Gatto LA, Fluck RR Jr - Alveolar mechanics in the acutely injured lung: role of alveolar instability in the pathogenesis of ventilator-induced lung injury. Respir Care, 2004;49:1045-1055

22. Antonaglia V, Peratoner A, De Simoni $L$ et al - Bedside assessment of respiratory viscoelastic properties in ventilated patients. Eur Respir J, 2000;16:302-308.

23. Ranieri VM, Brienza N, Santostasi S et al - Impairment of lung and chest wall mechanics in patients with acute respiratory distress syndrome: role of abdominal distension. Am J Respir Cit Care Med, 1997;156:1082-1091.

24. Musch G, Foti G, Cereda M et al - Lung and chest wall mechanics in normal anaesthetized subjects and in patients with COPD at different PEEP levels. Eur Respir J, 1997;10:2545-2552.

25. Ranieri VM, Giuliani R, Mascia L et al - Chest wall and lung contribution to the elastic properties of the respiratory system in patients with chronic obstructive pulmonary disease. Eur Respir J, 1996;9:1232-1239.

26. Jubran A, Laghi F, Mazur M et al - Partitioning of lung and chest-wall mechanics before and after lung-volume-reduction surgery . Am J Respir Crit Care Med, 1998;158:306-310.

27. Nava S, Rubini F - Lung and chest wall mechanics in ventilated patients with end stage idiopathic pulmonary fibrosis. Thorax, 1999;54:390-395.

28. Milic-Emili J - Expiratory flow limitation. Chest, 2000;117:(Suppl1):219S$223 S$.

29. Dhand R - Ventilator graphics and respiratory mechanics in the patient with obstructive lung disease. Respir Care, 2005;50:246-261.

30. Gottfried SB, Rossi A, Higgs BD et al - Noninvasive determination of respiratory system mechanics during mechanical ventilation for acute respiratory failure. Am Rev Respir Dis, 1985;131:414-420.

31. Bigatello LM, Davignon KR, Stelfox HT - Respiratory mechanics and ventilator waveforms in the patient with acute lung injury. Respir Care, 2005;50:235-245. 